J. Beerten, R. Eriksson, and D. Van Hertem, "A new approach to HVDC grid voltage control based on generalized state feedback," Proc. IEEE Power \& Energy Society General Meeting (PES GM) 2014, Washington DC, USA, Jul. 27-31, 2014, 5 pages.

Digital Object Identifier: 10.1109/PESGM.2014.6939418

URL:

http://ieeexplore.ieee.org/xpl/articleDetails.jsp?arnumber=6939418

(C) 2014 IEEE. Personal use of this material is permitted. Permission from IEEE must be obtained for all other users, including reprinting/ republishing this material for advertising or promotional purposes, creating new collective works for resale or redistribution to servers or lists, or reuse of any copyrighted components of this work in other works. 


\title{
A New Approach to HVDC Grid Voltage Control Based on Generalized State Feedback
}

\author{
Jef Beerten, Member, IEEE, Robert Eriksson, Member, IEEE, and Dirk Van Hertem, Senior Member, IEEE
}

\begin{abstract}
In this paper, a new approach to DC voltage control in a High Voltage Direct Current (HVDC) grid is introduced. In an HVDC grid, the power sharing after a converter outage can be influenced by the droop control values. However, when relying on a local feedback signal, the DC voltage drops in the system hamper a straight-forward definition of the power sharing. The use of a common DC voltage signal for the control can solve some of the problems. However, it disregards some of the benefits that are associated with the use of a local voltage control, such as the tendency of a controller using local voltage feedback signals to solve problems locally which can reduce the stress on the AC system. Furthermore, it provides no direct means to obtain different system responses for different converter outages. The control approach presented in this paper aims at improving the DC voltage droop control by combining the local voltage signal available at the converter terminals with remote voltage signals at different locations in the DC system by means of communication. The local voltage feedback control is used for a fast, reliable system response. The introduction of the remote voltage signals in the control allows to differentiate the system response for different converter outages. Simulation results show the validity of the proposed control scheme.
\end{abstract}

Index Terms-HVDC grids, voltage droop control, voltage source converter HVDC (VSC HVDC).

\section{INTRODUCTION}

$\mathbf{I}$ $\mathrm{N}$ recent years, the idea of building meshed systems based on High Voltage Direct Current (HVDC) technology has received considerable attention in the power engineering world [1]. Particularly the Voltage Source Converter (VSC) technology is a suitable candidate to build such a grid. The interest can be explained by the projected massive increase of volatile renewable energy sources in the generation mix. In the coming decades, these developments can give rise to the creation of an HVDC grid or a so-called supergrid, interconnecting different regions with their own generation and consumption patterns. The overall working principles and the control of such an HVDC grid have been studied in great depth over recent years. Especially the control of the DC voltage has received remarkable attention as it is as important to the DC system as the frequency is in an AC system. Similar to an AC system, a temporal imbalance between what is injected into the DC system and what is withdrawn from this system causes the DC voltage to deflect from its reference value. Similar as in AC

Jef Beerten is funded by a research grant from the Research Foundation Flanders (FWO). Robert Eriksson is funded by SweGRIDS. This work was supported by the EIT KIC InnoEnergy project Smart Power.

Jef Beerten and Dirk Van Hertem are with the Department of Electrical Engineering (ESAT), Division ELECTA, University of Leuven (KU Leuven), Kasteelpark Arenberg 10, bus 2445, 3001 Leuven-Heverlee, Belgium. (e-mail: jef.beerten@esat.kuleuven.be, dirk.vanhertem@esat.kuleuven.be). Robert Eriksson is both with the Electric Power Systems group (EPS) Royal Institute of Technology (KTH), Teknikringen 33, 10044 Stockholm, Sweden and the Center for Electric Power and Energy, Technical University of Denmark (DTU), Elektrovej, Kgs. 2800 Lyngby, Denmark. (e-mail: robert.eriksson@ee.kth.se). systems, a droop-based control, using a proportional control action can be used to distribute the control of the DC voltage over different converter stations. The majority of the control methods presented in the literature in recent years take this droop control as a starting point for the analysis and attention has been given to the design of the droop control itself [2] and the determination of droop values [3], [4].

There are, however, two main elements which make that the DC voltage control in a DC system is different when compared to the control of the frequency, its AC counterpart.

Firstly, contrary to the inertia in the rotating masses of the generators in an AC system, the DC system lacks a substantial amount of energy stored in the system. This makes that DC voltage variations following a disturbance are several orders of magnitude faster than $\mathrm{AC}$ frequency variations, and consequentially, so are the associated control actions: in case of a converter outage, the power changes resulting from the droop control actions appear to the $\mathrm{AC}$ system as a combination of instantaneous converter setpoint changes, which can trigger $\mathrm{AC}$ system transient instability. In [5], it is shown that different converter outages usually give rise to different preferred power sharing patterns when taking into account the connected AC system's response. However, this is hard to achieve with local feedback droop control or with a common voltage feedback signal in the droop control.

Secondly, the line resistive voltage drops in the DC system make that the DC voltage differs from point to point in a DC system and hence makes that the DC voltage measured locally is not a general control signal in the sense that the value depends on the system state and the power flow pattern in the DC grid. This is not the case for the frequency in an AC system. In [6], it was shown that the interaction of the droop voltage control with these line voltage drops makes that a trade-off exists between obtaining a predefined current sharing and limiting the voltage deviations after a contingency.

Using a common voltage signal or pilot voltage, as proposed in [7], could be considered to remove the above described influence, but it has a number of drawbacks. First, the performance of the overall control relies on communication. Second, it removes the local aspects of the voltage droop control observed in [6], [8]: converters that are electrically closer to the converter facing an outage tend to take a higher part of the power share in case of a local voltage feedback.

This paper aims at providing comprehensive insights in the advantages, drawbacks and limitations of the above described droop-based control options. Afterwards, a control methodology is introduced to overcome the drawbacks that both methods suffer from. The proposed control combines a fast voltage droop control based on a local feedback signal, with a control based on a voltage feedback of the different node voltages in the network. The overall idea is that the local 
feedback guarantees the scheme to operate fast and reliably, whereas the control based on remote voltage signals makes that the powers are shared in a predefined way, depending on which converter faces an outage. First simulation results confirm that the method can be a valuable candidate for future DC grid control, especially when more and more attention will be given to the preferred response of the interconnected hybrid AC/DC system.

\section{PRINCIPLES OF POWER SHARING IN DC SYSTEMS}

After a converter outage in an HVDC grid, the power deficit or surplus has to be compensated in order to stop the DC voltage respectively from decreasing or increasing in order not to damage the connected equipment or to stop the scheme from working. When using a droop control, various converters jointly alleviate the mismatch caused by the outage. The droop control can both be implemented based on current or power. In this paper, we have assumed that the control is current-based.

\section{A. Local voltage feedback signal}

When relying on a local voltage signal for the droop control, the current-voltage relation at a droop controlled converter station is given by

$$
I_{d c_{i}}=I_{d c, 0_{i}}-\frac{1}{k_{d c_{i}}}\left(U_{d c_{i}}-U_{d c, 0_{i}}\right),
$$

with $I_{d c_{i}}$ and $I_{d c, 0_{i}}$ respectively the actual and reference DC current at converter $i$.

The current sharing after a converter outage can be written in terms of the voltage droop constants in the different converters. Using (1), an outage of converter $i$ with a steady-state power injection of $I_{d c, 0_{i}}$ gives rise to the current redistribution in the converters which can be described as

$$
\begin{aligned}
& \Delta I_{d c_{i}}=-I_{d c, 0_{i}}, \\
& \Delta I_{d c_{j}}=I_{d c, 0_{i}} \cdot \gamma_{j},
\end{aligned}
$$

where $\gamma_{j}$ is the relative current sharing for converter $j$

$$
\gamma_{j}=\frac{g_{j} \Delta U_{d c_{j}}}{\sum_{\substack{k=1 \\ k \neq i}}^{m} g_{k} \Delta U_{d c_{k}}},
$$

with $\Delta U_{d c_{j}}=\left(U_{d c_{j}}-U_{d c, 0_{j}}\right)$ and the converter gain $g_{j}$ at converter $j$ defined as the inverse of the DC droop constant $k_{d c_{j}}$. It can be observed from these equations that the actual redistribution of the current after the contingency depends on the DC grid voltage profile after the fault, which impedes a straightforward analysis.

\section{B. Common voltage feedback signal}

As an alternative, a common voltage feedback signal can be used, as proposed in [7]. The droop control law then simplifies to

$$
I_{d c_{i}}=I_{d c, 0_{i}}-\frac{1}{k_{d c_{i}}}\left(U_{d c}{ }^{+}-U_{d c, 0}{ }^{+}\right),
$$

with $U_{d c}{ }^{+}$the common converter feedback signal and $U_{d c, 0}{ }^{+}$ its reference value. $U_{d c}{ }^{+}$can be the voltage at one of the converter buses or a combination thereof. Using a common voltage feedback signal removes the voltage dependence of the current sharing after an outage.

In case of a common voltage feedback signal used by all converters, as in (5), the relative current sharing $\gamma_{j}$ of converter $j$ after a contingency can be written as

$$
\gamma_{j}=\frac{g_{j}}{\sum_{\substack{k=1 \\ k \neq i}}^{m} g_{k}}
$$

It can be seen that the droop control law becomes independent of the voltages in the system. A disadvantage compared to a local voltage based droop control, is the need for communication.

\section{SECONDARY EFFECTS OF VOLTAGE DROOP CONTROL}

In case of a local voltage feedback signal, the voltages in the DC system influence the actual redistribution of the current after an outage. In [6], it was discussed that this influence cannot be eliminated without compromising the magnitude of the voltage deviations after an outage. In [8] it was found that the voltage dependency of the droop control makes that, when using similar droop settings at all the converters, a DC system has a tendency to solve deficits locally. This can be explained by the fact that the voltage in the DC system will normally change most considerably at the converter facing an outage, which influences the local voltage profile in the system more than it influences remote buses.

When building a droop control scheme using a common feedback signal for all outages, the dependency of the current sharing on the actual voltage profile is eliminated, as shown in (6). However, with this, the local effect of the droop control is also removed. In an HVDC grid, a common voltage feedback signal makes that all converters contribute similarly, independent of which converter faces an outage. From a system operator perspective, this can at a first glance be considered beneficial since, in this case, the droop values provide a direct and clear control variable to distribute the power contribution in case of an outage, similar to the frequency droop control in $\mathrm{AC}$ systems.

However, the fact that the local effect of the control action is lost can also be seen as an unwanted consequence. In [5], it was observed that different converter outages can give rise to different preferred power distributions from an AC grid pointof-view: a converter outage and the subsequent control actions undertaken by the converters give rise to a very fast change of the power flows in both the DC grid and the connected AC systems. This sudden change of power flows in the network can give rise to instable system behavior in the AC system. In this respect, it can be argued that the local aspects of the control action, which is inherently a part of local voltage feedback droop control, can be advantageous: in case a DC grid is spread over a large area, the deficit or surplus caused by a converter outage will give rise to a control action in which mainly nearby converters in the DC system alleviate the contingency. This especially holds when similar droop control settings are used for all converters. 


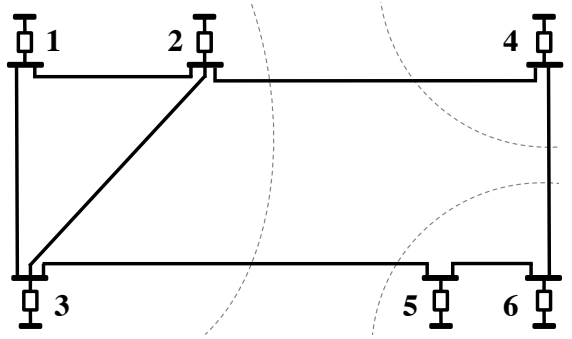

Fig. 1. A DC grid connected to an AC system with 3 distinct areas.

However, whether such a local control action is preferred from an $\mathrm{AC}$ system point of view largely depends on the internal structure of the underlying $\mathrm{AC}$ grids. This has been depicted conceptually in Fig. 1, where an overlay DC grid has been connected to an underlaying $\mathrm{AC}$ system with 3 distinctive zones with weak interconnections, represented by the dashed lines. In case the converters are both electrically nearby in the $\mathrm{AC}$ as well as in the DC system (e.g. converters 1, 2 and 3), such a local control action can be beneficial as the outage and the control actions will largely influence local power flows and leave power flows between remote areas (around converters 4, 5 and 6) largely unchanged. This could be beneficial in this case, as the three areas are remotely connected both in the $\mathrm{AC}$ and in the DC system. A more thorough study would be needed in such a case to make sure that the sudden change of power flows locally won't give rise to other problems.

On the contrary, in case the AC and DC system topology have a completely different structure and the electrical distances in both grids cannot directly be related, this local effect of the control might be an unwanted consequence. For example, in Fig. 1 converters 5 and 6 are electrically close to converter 4 at the DC side, but are remote in the AC system. In this case, a predominantly local power distribution at the DC side could trigger inter-area oscillations between corresponding remote $\mathrm{AC}$ areas, as discussed in [5].

In [6], it was shown that the effect of the droop control could be seen as an integral part of the system response by rewriting the power flow equations. The DC system equations can be written as

$$
\mathrm{Y}_{\mathrm{dc}} \boldsymbol{U}_{d c}=\boldsymbol{I}_{d c},
$$

with $\boldsymbol{U}_{\boldsymbol{d c}}$ the DC bus voltage vector, $\boldsymbol{I}_{\boldsymbol{d c}}$ containing the currents flowing into the DC system and $Y_{d c}$ the DC system admittance matrix.

Rewriting the voltage and current vectors

$$
\begin{aligned}
U_{d c} & =U_{d c, 0}+\Delta U_{d c}, \\
I_{d c} & =I_{d c, 0}+\Delta I_{d c},
\end{aligned}
$$

with the droop control law from (1),

$$
\Delta I_{d c}=-\mathrm{G} \Delta U_{d c}
$$

in which the gain matrix $G_{\text {out }}=\operatorname{diag}\left(\left[g_{1} \cdots \cdots g_{n}\right]\right)$. In case of a converter outage, these equations can be combined and applying the superposition principle

$$
\left(\mathrm{Y}_{\mathrm{dc}}+\mathrm{G}_{\mathrm{out}}\right) \Delta U_{d c}=I_{d c, \text { out }},
$$

with the modified gain matrix $G_{\text {out }}$ and the current outage vector $\boldsymbol{I}_{\boldsymbol{d c} \text {, out }}$ respectively defined as

$$
\begin{aligned}
\mathrm{G}_{\text {out }} & =\operatorname{diag}\left(\left[\begin{array}{lll}
g_{1} \cdots g_{i-1} & 0 & g_{i+1} \cdots g_{n}
\end{array}\right]\right), \\
\boldsymbol{I}_{\boldsymbol{d c}, \text { out }} & =\left[\begin{array}{lll}
0 \cdots 0-I_{d c, 0_{i}} & 0 & \cdots
\end{array}\right]^{T} .
\end{aligned}
$$

Rewriting the system equations this way, the droop control has been internalized as diagonal elements to the system admittance matrix by a modified admittance matrix

$$
Y_{d c}^{\prime}=Y_{d c}+G_{\text {out }} \text {. }
$$

In [6], it was shown that a trade-off exists between obtaining a predefined current redistribution and limiting the voltage deviations. The analysis in [6] was limited to the case where the current was redistributed in a uniform manner. The analysis can easily be extended to show that a similar trade-off exists when striving towards a more general redistribution similar to the one in (6). In other words, one would still have to make a trade-off when aiming to minimize the influence of the DC grid layout. However, as long as the redistribution is along the lines of the control reaction dictated by the DC line resistances, there are still means to make the current distribution from (4) to approximate the ideal distribution from (6).

On the contrary, it can be easily seen that the droop control as such, either when based on a local control signal or when based on a common control signal, provides very little means to distinguish between different converter outages, i.e. to obtain different current redistributions depending on which converter faces an outage. As discussed in [5], such a distinction could be beneficial from the AC system's point-ofview. As argued above, in case of a local droop control, there is an influence of the DC system layout, which makes that nearby converters tend to take a higher share than remote converters. In case of a common feedback signal, this distinction is not made since all converter outages are treated equal.

\section{Multiple INPUT FEEDbACK CONTROLlER DESIGN}

In the previous parts, it was explained that, especially when hybrid AC/DC system interactions are of interests, the standard voltage droop control principles suffer from a number of drawbacks. In this section, a new generalized droop control will be introduced, that uses multiple feedback signals.

Using the equations from the previous section, we redefine the gain matrix $G$ in (10) such that

$$
\mathbf{G}=\left[\begin{array}{cccc}
g_{11} & g_{12} & \cdots & g_{1 n} \\
g_{21} & g_{22} & \cdots & g_{2 n} \\
\vdots & \vdots & \ddots & \vdots \\
g_{n 1} & g_{n 2} & \cdots & g_{n n}
\end{array}\right]
$$

meaning that we can possibly use all converter voltage signals for a feedback in each converter droop-based controller.

In this multiple input generalized formulation, the modified gain matrix $G_{\text {out }}$ for an outage in converter 1 now becomes

$$
\mathrm{G}_{\text {out }}^{1}=\left[\begin{array}{cccc}
0 & 0 & \cdots & 0 \\
g_{21} & g_{22} & \cdots & g_{2 n} \\
\vdots & \vdots & \ddots & \vdots \\
g_{n 1} & g_{n 2} & \cdots & g_{n n}
\end{array}\right] .
$$


To determine the coefficients of the gain matrix, an objective function has been added aiming to limit the deviation from a predefined current distribution after any outage or

$$
\min f(\mathrm{G})=\sum_{i=1}^{n}\left\|\mathrm{G}_{\mathrm{out}}^{\mathrm{i}} \Delta \boldsymbol{U}_{d c}^{i}-\Delta \boldsymbol{I}_{d c}^{* i}\right\|^{2},
$$

with $\|\cdot\|$ the Euclidean norm and $\Delta I_{d c}^{* i}$ the reference values of the current injection changes at all converters for an outage of converter $i$. Contrary to the equal current distribution in [6], the setpoints have been chosen completely independent for the different converter outages.

The controller introduced in this article relies on local DC voltage feedback for a fast system response and uses the other converter voltage signals to distribute the current in a predefined way for each converter outage. Using a generalized state feedback approach provides the possibility to distribute the current in a predefined way which is not possible using only local feedback as discussed in the previous section.

The remote voltage signals introduce quite a significant time delay in the feedback which must be considered in the control design. A time delay limits the bandwidth of a feedback system which in the case of DC grids would make the dynamic response too slow when only relying on remote signals. This is since the delay sets a limit on the droop gains in (15), if fed back directly. The gains achieved solving (17) do not take any time delay or dynamic response into account as the formulation only corresponds to the steady-state solution. The diagonal elements, corresponding to the local feedback control, have to provide a fast system response to keep the overshot within limits. Appropriate local feedback gains can be set by choosing the diagonal elements in the gain matrix (15), after which the other gains are chosen to achieve the predefined current sharing. However, a solution given by (17) may give rise to an unstable system response due to the time delay associated with the control. This especially holds when the non-diagonal elements are large, i.e. when the current sharing is not in line with the DC system response dictated by the line resistances.

To come around this problem the remote feedback signals are pre-filtered through a low-pass filter. In this case, the remote signals only have an impact on the slow system response where the phase lag due to time delay is negligible. This implies that the non-diagonal elements do not have an impact on the bandwidth of the feedback system which is set by the local feedback gains. The pre-filtering can be performed by a first order filter where the filter parameters depend on the actual time delay and the non-diagonal gains.

\section{Simulation RESUlts}

To show the validity of the proposed scheme, simulations have been carried out on the 4-terminal $\pm 320 \mathrm{kV}$ DC grid from Fig. 2 [6], [9]. All converters have a rated output of 1200 MW. As an example, it has been assumed that converters 1 \& 3 and respectively $2 \& 4$ are better connected at the AC side and that there is therefore a clear preference for these nearby converters to share more power in case of an outage. The line resistances at the DC side are all of the same order

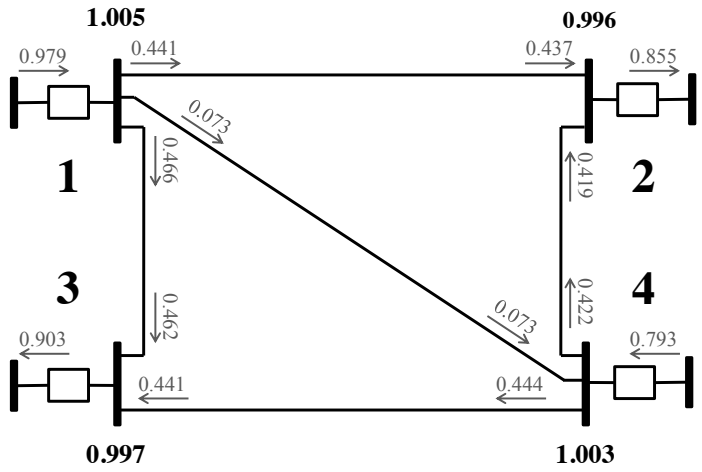

Fig. 2. Test-system - Power flow situation before the outage.

of magnitude [6]. The proposed relative current distributions in the different converters have therefore been chosen as

$$
\Delta \mathbf{I}_{\mathrm{dc}}^{* \text { rel }}=\left[\begin{array}{cccc}
-1 & 0.25 & 0.6 & 0.16 \\
0.25 & -1 & 0.2 & 0.66 \\
0.5 & 0.25 & -1 & 0.16 \\
0.25 & 0.5 & 0.2 & -1
\end{array}\right]
$$

which means that for example in case of an outage of converter 1 , converter 3 will take double the current as the two remaining converters. With the optimization routine from (17), and predefining the local feedback gains equal to 5 to obtain a satisfying local control response [9] to stabilize the voltage drop or rise as fast as possible, the obtained gain values are

$$
\mathrm{G}=\left[\begin{array}{cccc}
5 & 5.345 & 34.138 & 0.109 \\
1.002 & 5 & 0.060 & 53.0614 \\
27.299 & 0.051 & 5 & 6.627 \\
7.546 & 15.454 & 12.062 & 5
\end{array}\right]
$$

and a steady-state voltage deviation of

$$
\Delta \mathrm{U}_{\mathrm{dc}}=\left[\begin{array}{cccc}
-0.0083 & 0.0032 & -0.0004 & -0.0008 \\
-0.0025 & 0.0108 & -0.0009 & 0.0018 \\
-0.0009 & 0.0010 & 0.0082 & -0.0021 \\
-0.0019 & 0.0023 & 0.0018 & -0.0051
\end{array}\right]
$$

The current distribution shows a very good correspondence with the numbers from (18). Comparing the voltage deviations with those typically obtained using only local feedback signals [6], one can observe that the voltage deviations are reduced significantly. From (19), it can be observed that the requirement to primarily compensate the power at a particular converter causes the respective non-diagonal gains to increase significantly.

To show the validity of the scheme, dynamic simulations were carried out based on a linear DC system model in MATLAB. The inner converter current dynamics have been represented as a first order system with a time constant of 2 ms. A time delay of $20 \mathrm{~ms}$ has been assumed for the remote signals. In order to avoid instability in the controllers, a low pass filter with a time constant of $300 \mathrm{~ms}$ has been used to obtain a smooth response. When implemented in a real system, this constant can be changed to comply with the requirements from the point-of-view of the AC system stability. 


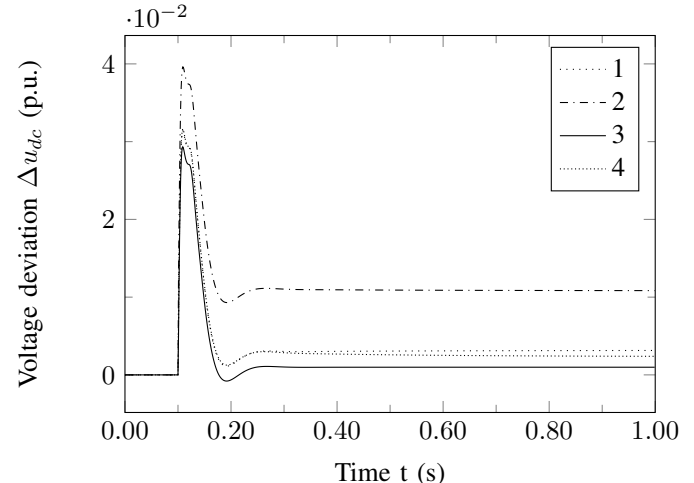

Fig. 3. Outage of converter $2-$ Voltage deviations $\Delta u_{d c}$ at the different converter terminals (Time simulations).

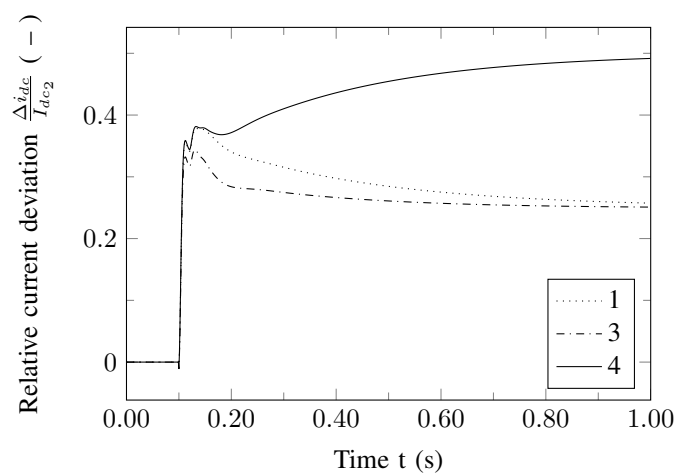

Fig. 4. Outage of converter 2 - Relative current sharing $\Delta i_{d c} / I_{d c_{2}}$ at the different converter terminals (Time simulations).

Figs. 3 - 6 show the time responses in case of an outage of converter 2. After the initial increase of the DC system voltage, which is compensated for immediately by the local voltage droop control, the slower control based on the remote voltage signals shifts the power flows as dictated by (18).

\section{CONCLUSION}

The novel voltage droop control scheme presented in this paper combines the advantages of a local droop control with the possibility to shift the powers to other converters based on the use of remote voltage signals for the control. The tendency of solving problems locally from a DC grid perspective, can but may not always be beneficial from the AC system stability point of view. In addition, depending on the grid layouts, a predominantly local response in the DC grid may not correspond to a local response as seen from the AC grid. Contrary to droop control using local voltages or a common voltage feedback, the proposed scheme provides the means to obtain different predefined system responses for different converter outages, which can be beneficial from an AC stability point-of-view. The scheme preserves the reliability of using local voltages for fast system response and combines it with information on the overall system state to set the controller gains to achieve a predefined system response. Simulation studies confirm that the presented control scheme allows to redistribute the powers in an arbitrary manner and that it gives rise to lower voltage deviations.

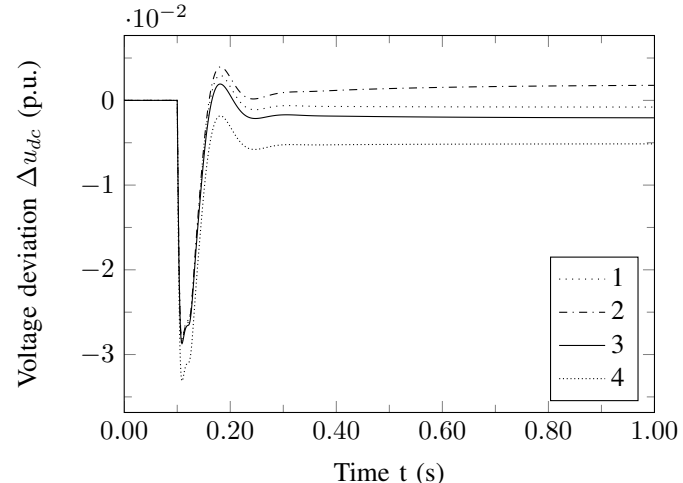

Fig. 5. Outage of converter $4-$ Voltage deviations $\Delta u_{d c}$ at the different converter terminals (Time simulations).

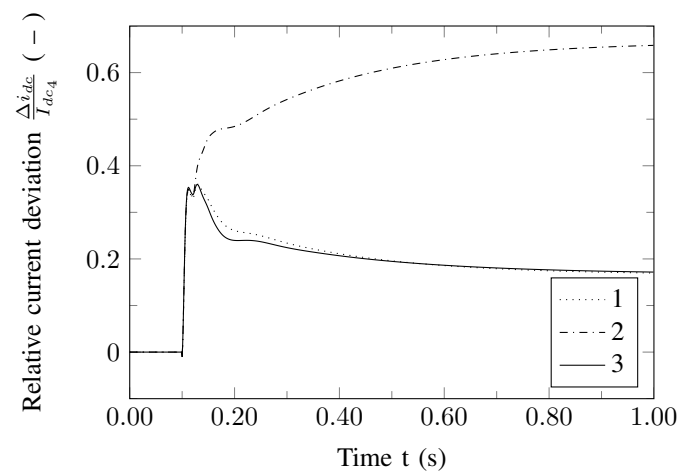

Fig. 6. Outage of converter 4 - Relative current sharing $\Delta i_{d c} / I_{d c_{4}}$ at the different converter terminals (Time simulations)

\section{REFERENCES}

[1] CIGRÉ Working Group B4-52, "HVDC grid feasibility study," Final Report, Apr. 2013.

[2] W. Wang, M. Barnes, and O. Marjanovic, "Droop control modelling and analysis of multi-terminal VSC-HVDC for offshore wind farms," in Proc. IET ACDC 2012, Birmingham, UK, Dec. 4-6, 2012, 6 pages.

[3] E. Prieto-Araujo, F. D. Bianchi, A. Junyent-Ferre, and O. GomisBellmunt, "Methodology for droop control dynamic analysis of multiterminal VSC-HVDC grids for offshore wind farms," IEEE Trans. Power Del., vol. 26, no. 4, pp. 2476-2485, 2011.

[4] L. Xu, B. W. Williams, and L. Yao, "Multi-terminal de transmission systems for connecting large offshore wind farms," in Proc. IEEE Power and Energy Society General Meeting - Conversion and Delivery of Electrical Energy in the 21st Century, 2008, 7 pages.

[5] R. Eriksson, J. Beerten, M. Ghandhari, and R. Belmans, "Optimizing DC voltage droop settings for AC/DC system interactions," IEEE Trans. Power Del., 2013, 8 pages, accepted for publication.

[6] J. Beerten and R. Belmans, "Analysis of power sharing and voltage deviations in droop-controlled DC grids," IEEE Trans. Power Syst., vol. 28, no. 4, pp. 4588-4597, 2013.

[7] B. Berggren, R. Majumder, C. Sao, and K. Lindén, "Method and control device for controlling power flow within a dc power transmission network," Patent WIPO International Publication Number WO 2012/000 549A1, International Filing Date: Jun. 30, 2010.

[8] T. M. Haileselassie and K. Uhlen, "Impact of DC line voltage drops on power flow of MTDC using droop control," IEEE Trans. Power Syst., vol. 27, no. 3, pp. 1441-1449, Aug. 2012.

[9] J. Beerten and R. Belmans, "Modeling and control of multi-terminal VSC HVDC systems," Energy Procedia, vol. 24, pp. 123-130, Aug. 2012, selected papers from Deep Sea Offshore Wind R\&D Conference, Trondheim, Norway, Jan. 19-20, 2012. 